\title{
Development and Control of a 5-Axis Reconfigurable Machine Tool
}

\author{
Z. M. Bi \\ Department of Engineering, Indiana University—Purdue University Fort Wayne, Fort Wayne, IN 46805-1499, USA \\ Correspondence should be addressed to Z. M. Bi, biz@ipfw.edu
}

Received 13 January 2011; Revised 19 March 2011; Accepted 4 May 2011

Academic Editor: Yangmin Li

Copyright () 2011 Z. M. Bi. This is an open access article distributed under the Creative Commons Attribution License, which permits unrestricted use, distribution, and reproduction in any medium, provided the original work is properly cited.

The development of a hybrid reconfigurable machine tool has been introduced. The machine tool consists of a tripod-based parallel kinematic machine (PKM) module with three degrees of freedom (DOF) and a serial linear $X-Y$ table with two DOF. The PKM is installed on a gantry system which is capable of reconfiguring its position and orientation. In the design of tripod-based parallel mechanism, a passive link is used to enhance the stiffness and increase the working load. To avoid the buildup of the heat of the extensive actuation, three joints are actuated via the actuators with a constant length. The geometries of the PKM have been optimized for the best and highest accuracy. In this paper, its control system and the prototyping development are focused. An open architecture is applied, the control methodologies are developed and validated, and the corresponding software tools have been implemented for the software reconfiguration of the control system.

\section{Introduction}

The globalization of the manufacturing environment brings uncertainties and turbulences of customers' requirements and manufacturing resources. Reconfigurable manufacturing system (RMS) paradigm becomes an effective means to increase the competitiveness and adaptability of manufacturing systems. An RMS consists of modular components that can be arranged to meet the machining needs (reconfiguration) with minimal or nonrecurring cost. The ability to reconfigure machines allows a quick ramp-up and robustness to adapt various changes in a dynamic production environment. Production machinery is usually specialized and very capital intensive. Other benefits of an RMS is its potential to reduce the unit cost of machining; the same system can serve for different tasks and, thus, increase the utilization of resources, and the machine modules with a certain volume make it possible to be fabricated and assembled in a mass production mode.

The basic strategy to make a system reconfigurable is the modularization of system. Under modularized architecture, the system is formed from a set of modules. These modules can be connected one with another interchangeably, and various system configurations can be generated by using different types of modules, changing the number of modules, or changing the topology of module connections. Each configuration can be specially designed to meet the given requirements optimally [1]. System modularization has been well recognized in machine tool industry. Modular machine tools have been on the market for a few of decades. The international standards became available to machine modules in the seventies [2]. Researchers have made continuous effort in improving modular machine tools. For example, Hannover University initialized a project called Modular Synthesis of Advanced Machine Tools (MOSYN) to support the design of customer-specific configurations of modular machine tools [3]. Nevertheless, it is worth to note that the primary objective of the machine-tool builders to modularize their products is to produce the variants; on other hand, machine-tool users still purchase the systems with a specific configuration, and these systems are rarely reconfigured after their installments.

Differing from traditional modular machine tools, the reconfigurable machine tool at the University of Michigan aims at modularity, integrability, customization, convertibility, and diagnosability. The system is designed to reconfigure 


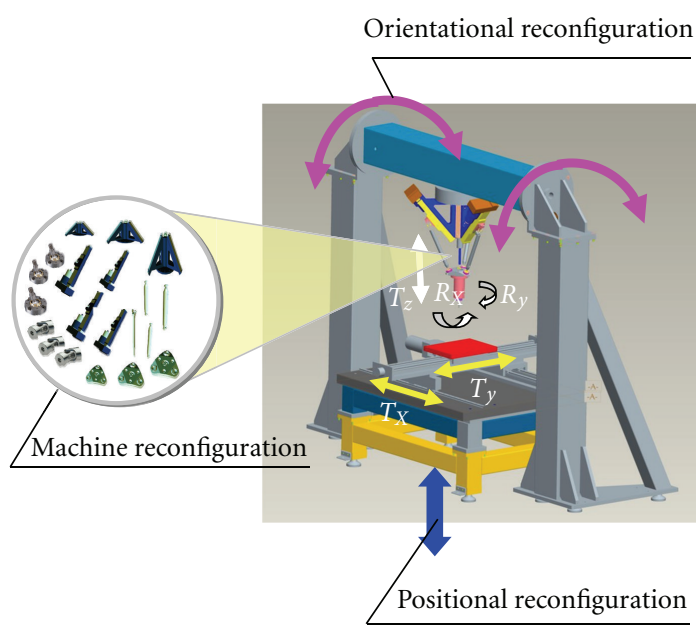

(a) Virtual model

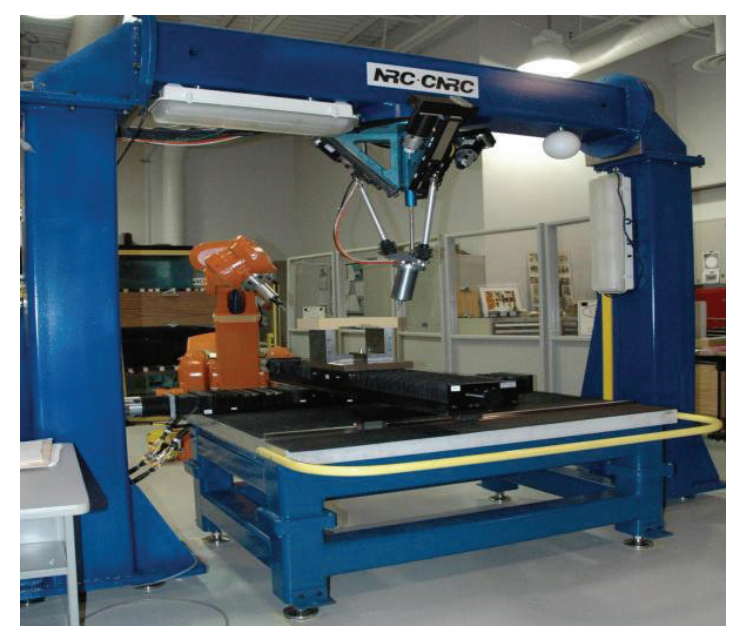

(b) Physical model

FIgURE 1: Virtual and physical models of 5-axis machine tool.

frequently in a dynamic environment [4-6]. Unfortunately, the design concept was not fully implemented, and some obstacles slowed down their research and development [7]. The reconfigurable Parallel Kinematic Machine (PKM) is another alternative to reconfigure machine tools [8-10]. Theoretically, PKMs have the potentials to achieve higher speed, higher accuracy, and deal with a heavier load. However, the characteristics of the PKMs have not been well studied. Most of the commercially available PKMs are highcost machines that provide lower accuracy than conventional machine tools [7, 11]. More studies are on demand to develop competitive PKMs. For example, Zhao et al. [12] introduced a new parallel simulator with six degrees of freedom (DOF); this new PKM adopts redundant actuations to increase the capacity to resist external load and prolong the actuators' life time. Redundancy of inverse kinematics for a redundant robot is often addressed by the pseudoinverse method; Wang et al. expanded this method as a resolution for the redundant relations at velocity and acceleration levels, so called closed-loop inverse kinematics algorithm was proposed to control a 7-DOF redundant PKM [13]. Yun and Li developed the stiffness models for the flexure hinges [14]; their work has its significance in predicting the deflections and improving the precision of PKMs. Researchers have continued in making progress on reconfigurable robotic systems. For example, a type of square-cubic-cell module has been designed as the basic element to build various robotic configurations for different application. The targeted configurations can be open loop, closed loop, or a combination of both [15].

To develop a cost-effective PKM machine tool, the tripod-based PKM is focused due to its two significant merits, that is, the versatility and the benefits of a closedloop mechanism. Tripod-based PKMs are the most versatile one among the existing PKMs. A tripod-based PKM typically possesses three DOF. Depending on its structure, it can produce an end-effector motion with the pure rotation, the pure translation, or a combination of rotation and translation.
Tripod-based PKMs can perform many different machining operations since the majority of the machining operations require a motion with five or less DOF [9]. A tripod-based PKM can be used as a stand alone machine or a module in a system. As a stand-alone machine, it can be a substitution of a traditional machine tool with three or less DOF. As a system module, it can be combined with other actuated modules or subsystems.

A new tripod-based machine with a passive leg has been proposed by the author, and the details of design methodologies and assistive design toolbox have been reported $[16,17]$. In this paper, the control method and implementation of this machine tool will be focused. The remainder of the paper is organized as follows. In Section 2, the hybrid machine structure is introduced, the parallel structure for positioning is specially examined, and its kinematic model is overviewed. In Sections 3 and 4, the requirements of system control are discussed, and control architecture is proposed accordingly. The control model is developed for the machine tool, and the implementation of system control is discussed. In Section 5, a calibration approach for system reconfiguration is introduced. Finally in Section 6, the research works presented in this paper are summarized.

\section{Structure of Five-DOF Reconfigurable Machine Tool}

Figures 1(a) and 1(b) have illustrated the virtual model and physical model of the new hybrid reconfigurable machine tool, respectively. This machine tool consists of three modules: a tripod tool, an $X-Y$ table, and a reconfigurable arch. This machine tool is hybrid, the positioning structure is a tripod-based PKM with three DOF, and the orienting structure is a serial wrist with two DOF.

The tripod-based PKM is the most important module. The modularized tripod-based PKM includes three linear actuators; these actuators can be reconfigured and integrated 
with the customisable base and end-effector platforms. The geometries of the base and end-effector platforms can change the behaviours of the parallel structure significantly. The tripod-based PKM structure is expected to provide the rotations about $X$ axis and $Y$ axis and the translation along the $Z$ axis. Reconfigurability of the machine tool can be further enhanced by the adjustments of the $X-Y$ table and reconfigurable arch. Both of them are reconfigurable. The $X-Y$ table provides various motion ranges of translation along $X$ and $Y$ axes, and its height along the $Z$ axis can be reconfigured offline. The arch is an assembly of three components: two vertical columns and a horizontal beam. The orientation of the horizontal beam can be reconfigured to accommodate the changes of location and orientation of the working volume.

The variations of the reconfigurable machine tool are from some customerized modules in the system. The dimensions of these modules such as binary legs and the platforms can be customized based on the given task. The adjustable arch and the $x-y$ table allow for many configurations to meet different manufacturing requirements, for example, changing from a vertical configuration at various discrete angles towards a horizontal configuration, if required. System control software is also reconfigurable to represent a correct kinematic behaviour of the reconfigured machine tool. The key to this reconfiguration is the quick verification of the new machine geometry. Instead of lengthy calibration of the machine, only a quick measurement of a few reference points is required, and the calibration of the machine is accomplished in the controller internally.

\section{Tripod-Based PKM}

The tripod-based PKM of the machine tool is parameterized in Figure 2. There are three platforms: base platform $B_{1} B_{2} B_{3}$, middle platform $M_{1} M_{2} M_{3}$, and end-effector platform $E_{1} E_{2} E_{3}$. The base platform is fixed on the ground. The middle platform is to support guide-way $B_{i} M_{i}$ of active leg $D_{i} E_{i}$. The end-effector platform is to mount a tool or gripper. A passive link is installed between the middle platform and the end-effector. Active leg $D_{i} E_{i}$ is connected to the endeffector platform by a spherical joint at $E_{i}$, and to the slide of the active prismatic joint by a universal joint at $D_{i}$. The passive leg is fixed on the middle platform at one end, and connected to the end-effector platform by a universal joint at the other end. The parameters for the description of the tripod machine tool are as follows: the angle $\alpha_{i}(i=1,2,3)$ between $O_{b} B_{i}$ and $\mathbf{x}_{b}$, the angle $\beta_{i}(i=1,2,3)$ between $O_{e} E_{i}$ and $\mathbf{x}_{e}$, the radius of the base platform $l_{b}$, the radius of the end-effector platform $l_{e}$, the direction of a guide-way $\gamma$, and the length of an active leg $l_{i}$.

The DOF of the tripod-based PKM can be calculated as

$$
f=6 \times(n-1)-\sum_{i=1}^{5}(6-i) \times j_{i}=3,
$$

where $f$ : DOF of the mechanism, $n$ : the number of rigid bodies including the base, $j_{i}$ : the number of joints with $i$-DOF.

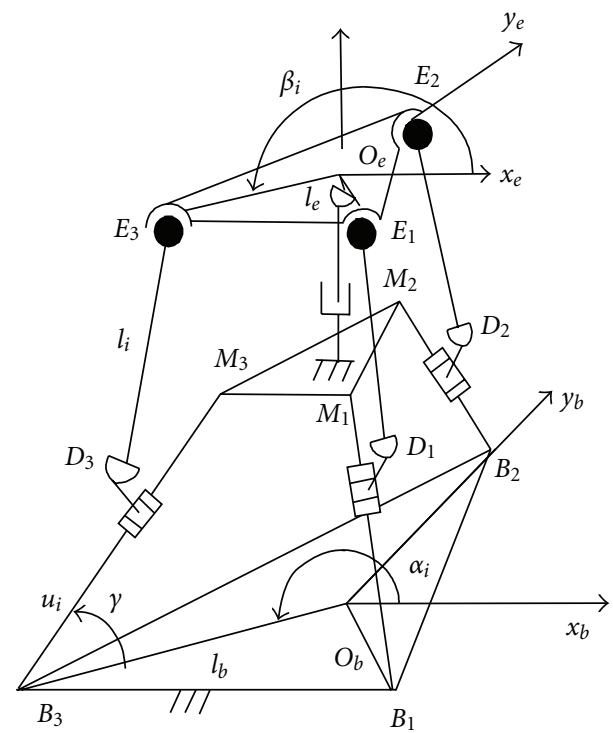

FIGURE 2: Parametric description of tripod-based PKM structure.

Note that the tripod-based PKM has 8 rigid bodies, 3 prismatic joints, 3 rotational joints, and 3 spherical joints.

The complete description of the motion of a rigid body requires three rotational parameters $\left(\theta_{x}, \theta_{y}, \theta_{z}\right)$ and three translational parameters $\left(x_{e}, y_{e}, z_{e}\right)$. However, $x$ - and $y$ translations and $z$-rotation are eliminated because of the passive link. Therefore, the motion of the end effector can be fully described by three independent variables $\theta_{x}, \theta_{y}$, and $z_{e}$, where $\theta_{x}$ and $\theta_{y}$ are the rotations about $x$ and $y$, and $z_{e}$ is the translation along $\mathbf{z}$. The posture of the end effector tool is represented by

$$
T_{e}^{b}=\left[\begin{array}{cc}
R_{e} & P_{e} \\
0 & 1
\end{array}\right]
$$

where $T_{e}^{b}$ is the posture of the end-effector with respect to $\left\{\mathbf{O}_{b}-\mathbf{x}_{b} \mathbf{y}_{b} \mathbf{z}_{b}\right\}$.

$$
R_{e}=\left[\begin{array}{ccc}
\cos \theta_{y} & 0 & \sin \theta_{y} \\
\sin \theta_{x} \sin \theta_{y} & \cos \theta_{x} & -\sin \theta_{x} \cos \theta_{y} \\
-\cos \theta_{x} \sin \theta_{y} & \sin \theta_{x} & \cos \theta_{x} \cos \theta_{y}
\end{array}\right]
$$

is the rotational matrix of the end effector.

$P_{e}=\left[\begin{array}{lll}0 & z_{e}\end{array}\right]^{T}$ is the central position $\mathbf{O}_{e}$ of the end effector.

When the required position of the end-effector tool is specified, inverse kinematics is to find the joint displacements. Assume that the joint displacement of active prismatic joint $i$ is $u_{i}$, it can be derived based on the fact that the length of an active link is fixed, thus,

$$
u_{i}=\frac{-k_{b} \pm \sqrt{k_{b}^{2}-4 k_{c}}}{2}
$$




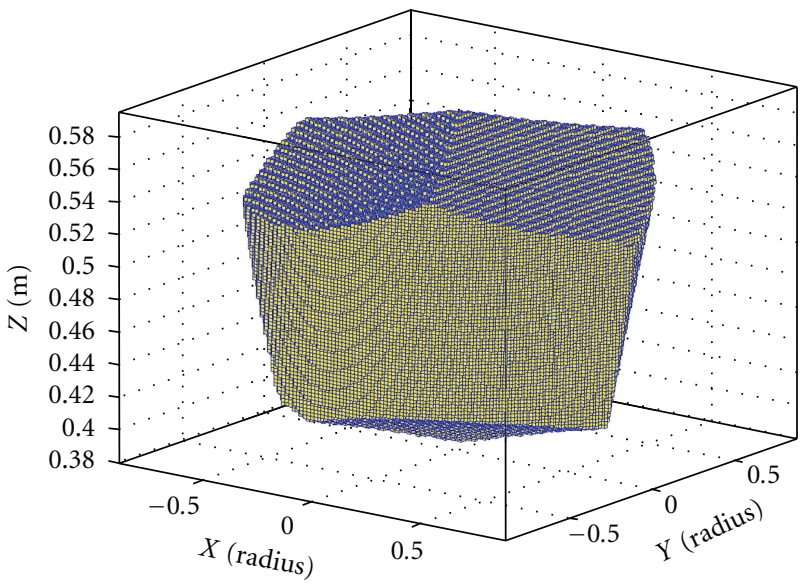

(a) Task workspace

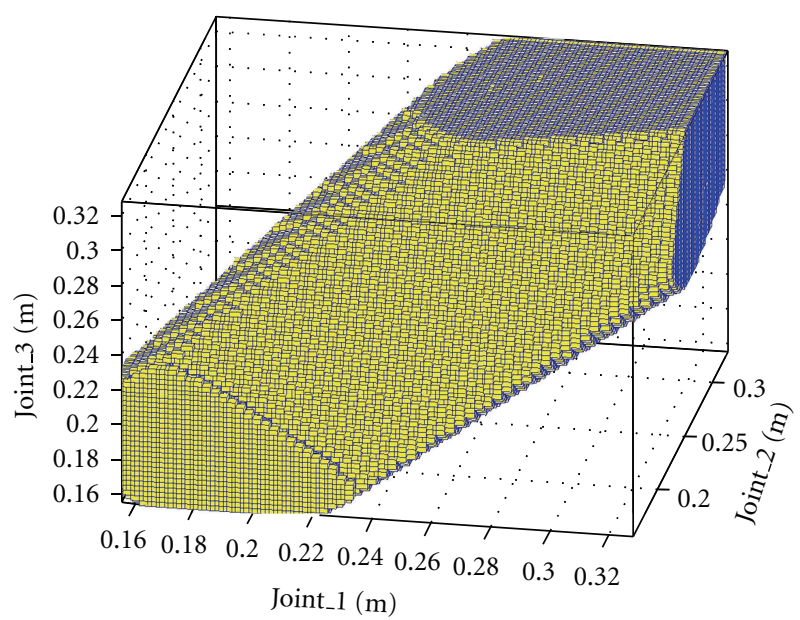

(b) Joint workspace

FIGURE 3: Task and joint workspace.

where

$$
\begin{aligned}
k_{b}= & 2\left(\cos \gamma\left(x_{e_{i}}^{b} \cos \alpha_{i}+y_{e_{i}}^{b} \sin \alpha_{i}-l_{b}\right)-z_{e_{i}}^{b} \sin \gamma\right), \\
k_{c}= & \left(x_{e_{i}}^{b}\right)^{2}+\left(x_{e_{i}}^{b}\right)^{2}+\left(x_{e_{i}}^{b}\right)^{2}+l_{b}^{2}-l_{i}^{2} \\
& -2 l_{b}\left(x_{e_{i}}^{b} \cos \alpha_{i}+y_{e_{i}}^{b} \sin \alpha_{i}\right) .
\end{aligned}
$$

Note that the solution of $u_{i}$ from (4) should be examined to ensure none of active or the passive joints exceeds its motion limit.

3.1. Task and Joint Workspace. Workspace is an important characteristic to describe the performance of a PKM. Workspace is usually defined as the set of reachable points of the tool center point (TCP) in the Cartesian coordinate system. It can be determined by the inverse kinematic solver numerically, an example of task workspace has been illustrated in Figure 3(a) for this machine tool.

Motion ranges of joints have usually been given for the reconfigurable machine tool. However, whether or not a joint could be moved freely over its motion range depends on the structure of the PKM and the configuration of the PKM at a certain position. The PKM is to transfer joint motions into the motion of TCP. A new concept called joint workspace is proposed to measure the efficiency of this transformation [18]. The solver to forward kinematics is needed to acquire the joint workspace of a PKM. We have formulated the forward kinematic problem as a polynomial equation whose coefficients can be determined automatically. As shown in Figure 3(b), an example of the joint workspace has been illustrated for the prototyped PKM.

3.2. Deflection under External Loads. Deflection of the rigid bodies under an internal or external load causes positional and orientational errors of the TCP. Stiffness is defined to measure the capability of a PKM to resist the deflection. System stiffness along all of the six Cartesian motion axes is evaluated based on a kinetostatic model. Figure 4 shows an example of the stiffness distribution along all of the six motion axes, respectively, for a cross-section where all of the active joints are in the middle of their motion ranges.

3.3. Estimation of Driving Forces. Since all of the existing PKMs use kinematic control, a dynamic model of a PKM becomes less important. However, a typical robotic task not only has the requirements of a given motion trajectory but also the requirements of a working load. A dynamic model will be needed to determine how an external load is carried out by the driving forces at the actuators. At an extreme case when the external load becomes critical, the dynamic model is required to estimate if an actuated joint has the sufficient driving force to resist the external load. A dynamic model based on Newton-Euler approach has been developed. The modeling procedure is as follows: (i) the displacements, velocities, and accelerations of the joint motions are obtained from an inverse kinematic model under the given end-effector motion; (ii) the velocities and accelerations of the joints and mass centers of all bodies are derived sequentially; (iii) the Newton's law and Euler's equation are applied to calculate inertia forces/moments, and the equilibrium equations are defined for each of bodies; (iv) the dynamic model of the system is assembled from the equilibrium equations of the bodies; (v) the dynamic model is solved to get the forces/torques of joints. The driving forces of the actuated joints, as well as the reaction forces on any of passive joint(s), can be analyzed. Figure 5 has shown an example of the driving forces corresponding to a given TCP motion.

\section{Control Architecture and Model}

After system reconfiguration, the machining operation needs to control the components including three linear actuators, the spindle on the end-effector tool, and two linear actuators on the $X-Y$ table. Since the reconfigurable machine is a 


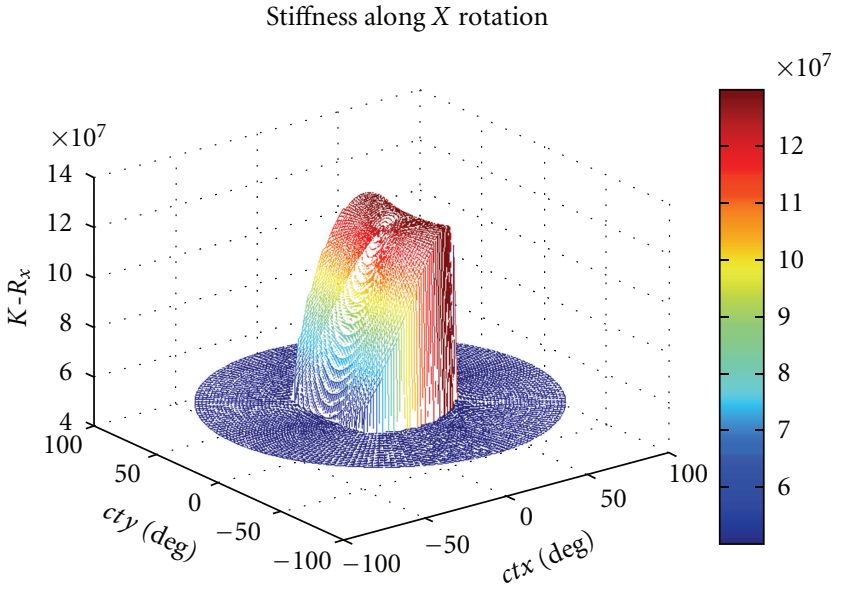

(a) $R_{x}$

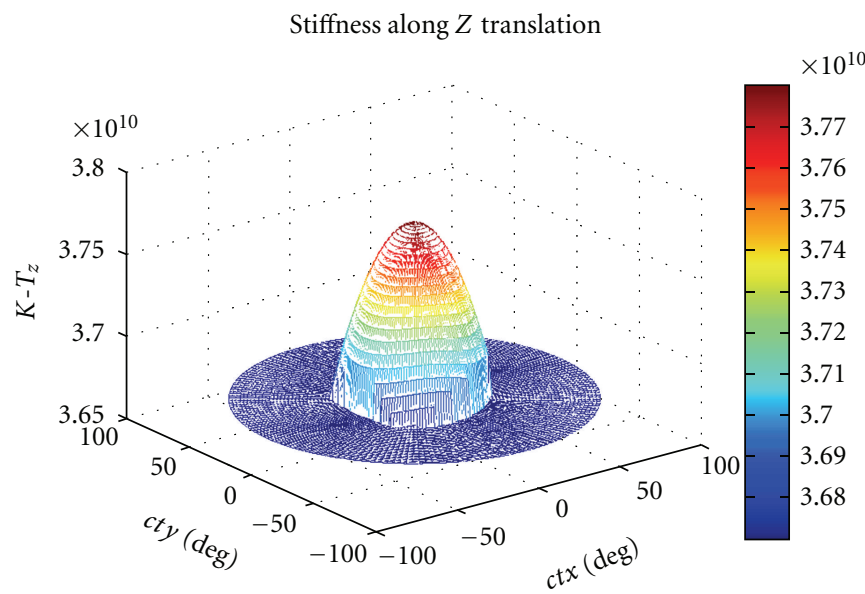

(c) $T_{z}$

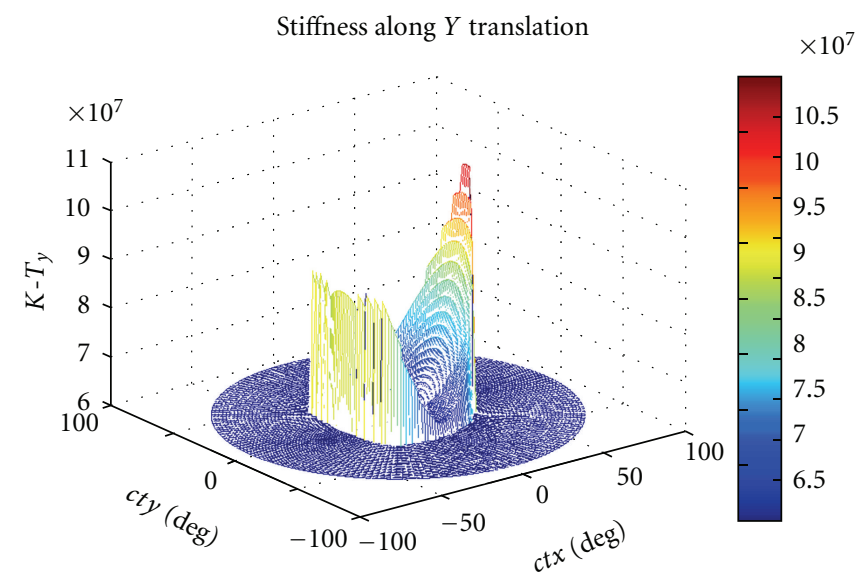

(e) $T_{y}$

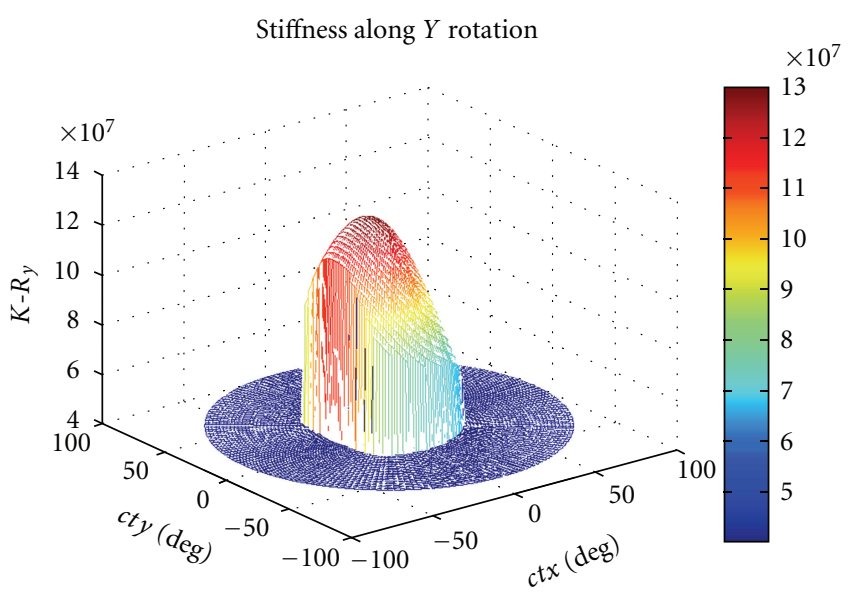

(b) $R_{y}$

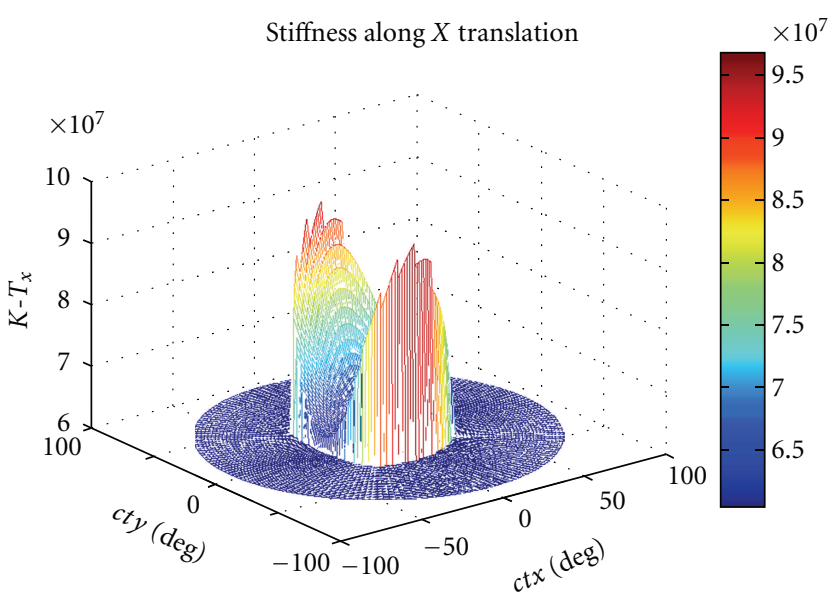

(d) $T_{x}$

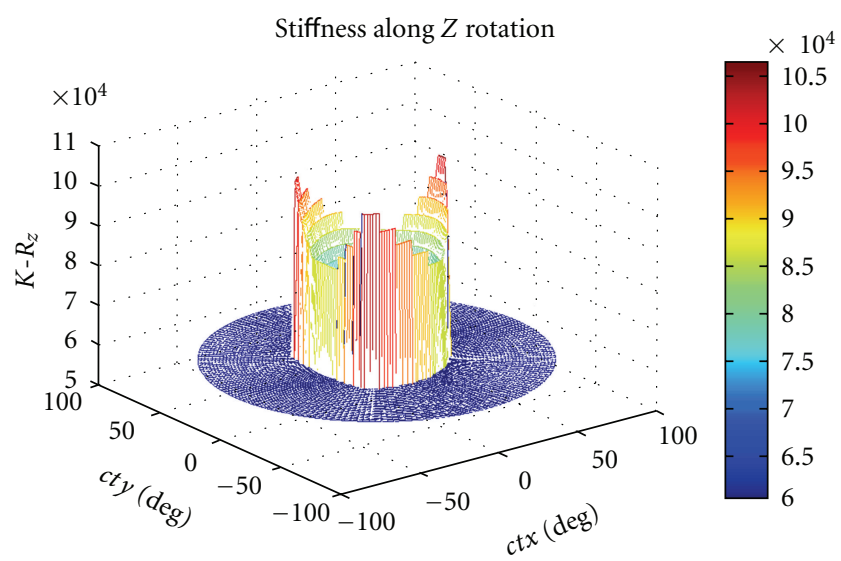

(f) $R_{z}$

FIGURE 4: Stiffness over the middle cross-section of task workspace.

hybrid, the motions of these components are coupled when the motion of the task is given.

The control architecture for the reconfigurable machine tool is shown in Figure 6. The motion of the tripod-based
PKM links all of the control expectations of the system components including the tripod tool, the spindle, and the $X-Y$ table. The determination of the feed depth is based on the $Z$ translations of both from the spindle and the tripod 


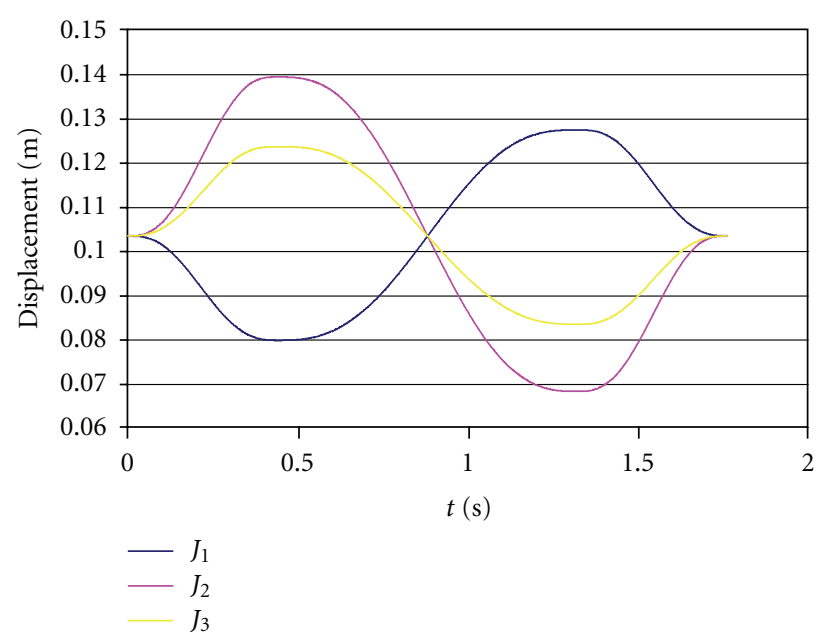

(a) Displacements of actuated joints

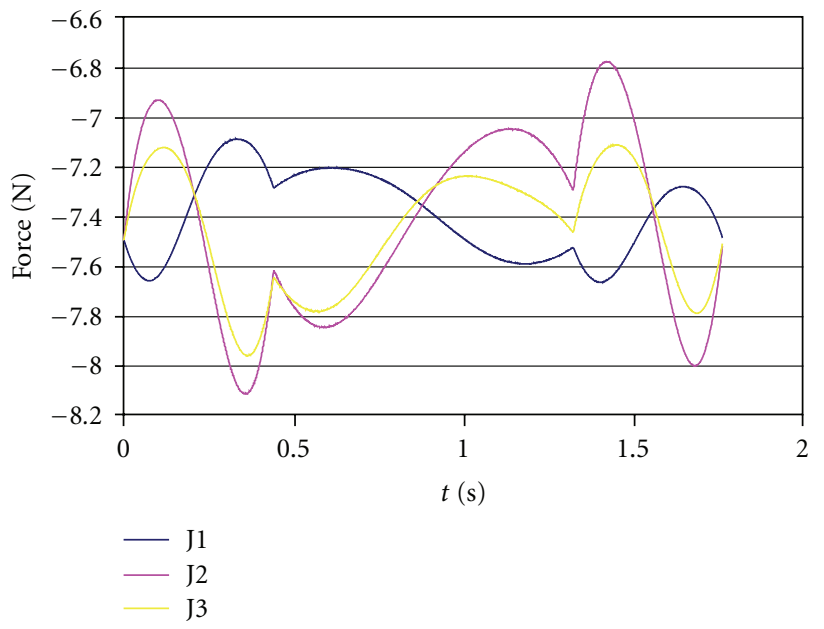

(b) Forces of actuated joints

FIGURE 5: Estimation of driving forces based on a dynamic model.

tool. The coordinates of the $X-Y$ table must compensate the $X$ and $Y$ displacements caused by the rotation of the spindle about $X$ and $Y$.

When the machine is set at the vertical tool orientation, the machine and its control behave like a regular 5-axis machine with a tilting and pivoting tool head. The tool path is generated in the Cartesian space, and postprocessed with a generic CAM package for a 5-axis machine. The operation, programming, and control of the tripod module are transparent to the user. The last three components of a machine positioning command, $Z, A$, and $B$ are used to represent the displacements of three actuated joints from the inverse kinematics.

When the machine is configured differently from the vertical tool axis, additional transformations have to be carried out. For the calculations of general transformations, the homogeneous coordinates are used, which were originally developed for robot manipulator control [18]. Note that an offline simulation of machining process is helpful. When the head angle is getting closer to the horizontal, the working envelope is degenerating. In the horizontal position, the machine "looses" a degree of freedom, the $Z$ tool axis movement is in the Table's $X-Y$ plane.

Figure 7 has shown the coordinate frames of the "Table", or the $X-Y$ table, and the "Tripod" in a general configuration. The "Tripod" is rotated along its $X$ axis by $\theta$ angle and translated by $x_{\mathrm{TR}}, y_{\mathrm{TR}}$, and $z_{\mathrm{TR}}$ respectively. The transformation matrix ${ }^{T} \mathbf{T}_{\mathrm{TR}}$ from the "Table" frame to the "Tripod" frame is given as follows:

$$
{ }^{T} \mathbf{T}_{\mathrm{TR}}=\left[\begin{array}{cccc}
1 & 0 & 0 & x+x_{\mathrm{TR}} \\
0 & \cos \theta & -\sin \theta & y+y_{\mathrm{TR}} \\
0 & \sin \theta & \cos \theta & z_{\mathrm{TR}} \\
0 & 0 & 0 & 1
\end{array}\right],
$$

where $x$ and $y$ represent the motion commands for the $X$ and $Y$ axes.
The NC part program in this case is given in the $x^{\prime}-y^{\prime}-z^{\prime}$ coordinate system, since machining conventions require that the tool axis is always the $Z$ axis. To control the $X$ and $Y$ axis coordinates, the calculation of the inverse kinematics, "Tripod" to "Table" transformation ${ }^{T} \mathrm{~T}_{\mathrm{TR}}{ }^{-1}$, is required.

\section{System Calibration}

The fabrication and assembly of the machine components bring geometric errors, and these errors reduce the accuracy of the machine tool. To achieve a higher precision of the reconfigurable machine tool, the calibration is required after a reconfiguration is performed. The calibration includes both the geometric confirmation of the machine reconfiguration as well as the verification of the Tripod kinematics. Conventional machine tools are usually calibrated when they are delivered, assembled, and commissioned. The process is expensive, time consuming, and requiring specialized equipment and personnel. As those machines do not change their configuration, the calibration process is repeated only seldom, after machine overhauls or at required prescheduled intervals. Reconfigurable machines, on the other hand, may change their configurations based on the quickly changing production requirements. While making the discrete configuration positions repeatable and accurate by mechanical means, it is simpler to adjust the machine, measure, and calibrate in software. The emergence of some high-end optical tracking devices such as FARO Laser Tracker makes it possible to have quick and accurate volumetric measurement.

An optimal calibration methodology for the kinematic model has been developed. The basis of the methodology is to exploit the least error sensitive regions in the workspace. An error model has been developed that takes into consideration all of the geometric errors of modular components. Analysis of this error model has shown that the error mapping from the geometric errors to the pose error of the PKM depends on the Jacobian matrices. The calibration 


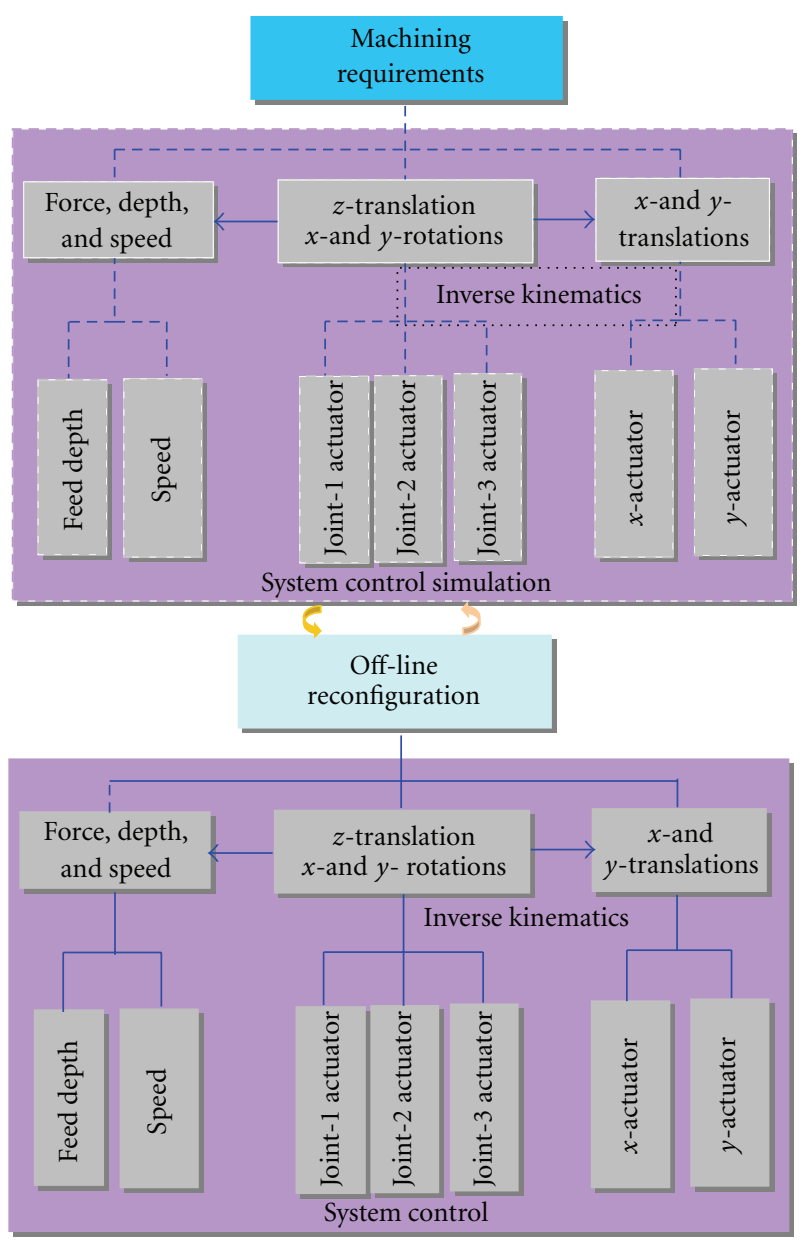

FIgure 6: Control Architecture of Reconfigurable Machine Tool.

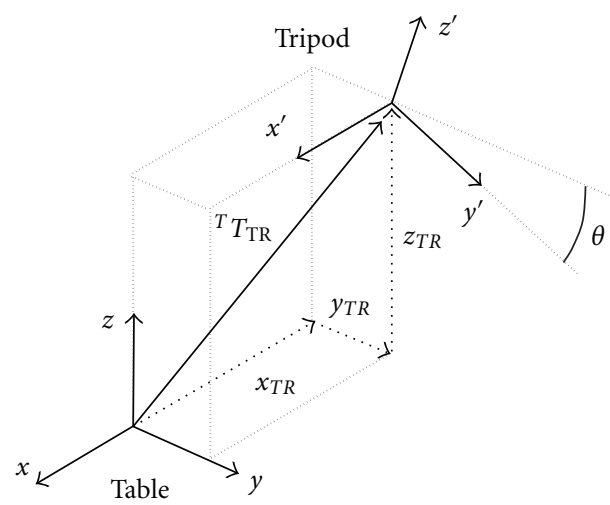

Figure 7: The coordinate system of the tripod machine tool.

routine has been coupled with an automatic alignment system that uses a laser-based measurement system. To maintain the accuracy of the robotic operation in the working volume, one of the requirements is to keep the correct ${ }^{T} \mathbf{T}_{\mathrm{TR}}$ coordinate transformation matrix. Figure 8 has shown the methodology to derive this matrix. Both of the "Table" and "Tripod" reference frames are established with respect to the same reference origin in the "World" frame; this origin is

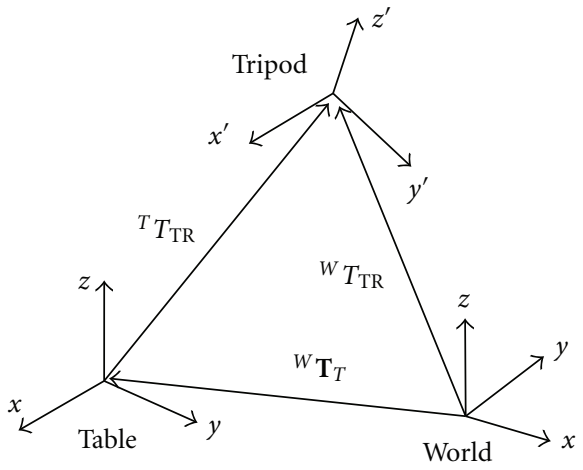

Figure 8: Calibration of tripod machine tool.

coincident to the origin of the optical measurement system. ${ }^{T} \mathbf{T}_{\mathrm{TR}}$ then can be defined by

$$
{ }^{T} \mathbf{T}_{\mathrm{TR}}={ }^{W} \mathbf{T}_{T}{ }^{-1} \cdot{ }^{W} \mathbf{T}_{\mathrm{TR}},
$$

where ${ }^{W} \mathbf{T}_{T}$ and ${ }^{W} \mathbf{T}_{\mathrm{TR}}$ are the reference frames of the "Table" and the "Tripod" with respect to the "World" frame.

\section{Control Implementation}

The control system for the reconfigurable machine tool is implemented on open architecture control (OAC) architecture to adopt new features or modify existing functions in a control system quickly and freely. The OAC is built on the ORTS platform [19], which is a heterogeneous, multiprocessor real-time system, capable of handling the PC hardware, and more than one DSP processor. The hardware platform includes an industrial PC equipped with a digital signal processor (DSP) board and motion control interface cards. The interface has the connections to the machine tool to control auxiliary axes.

The software architecture for the control system is depicted in Figure 9. The distribution of functionalities is based on the real-time requirements of the individual tasks. The DSP part (for which ORTS is the native operating system) handles the hard real-time requirements. The PC part of ORTS runs under the Windows NT operating system, and it only meets soft real-time needs. On the DSP, there are tasks for path generation, servo loop control, and the machine logic interface, while on the PC the user interface is realized.

The ORTS provides a rich set of intertask, and interprocessor communication means to implement complex realtime systems. Controlling the tripod-based PKM requires new functionalities in the $\mathrm{OAC}$ because of its unique kinematics; the actuators control the motion of the PKM in joint coordinates, while the user or machining task is described in a Cartesian work space. For smooth and accurate control, these transformations of coordinates are performed at the rate of the servo sampling time, that is, every set point from the path generator are transformed before presenting it to the servo loops. Along with the motion transformation, geometrical error checks are also performed 


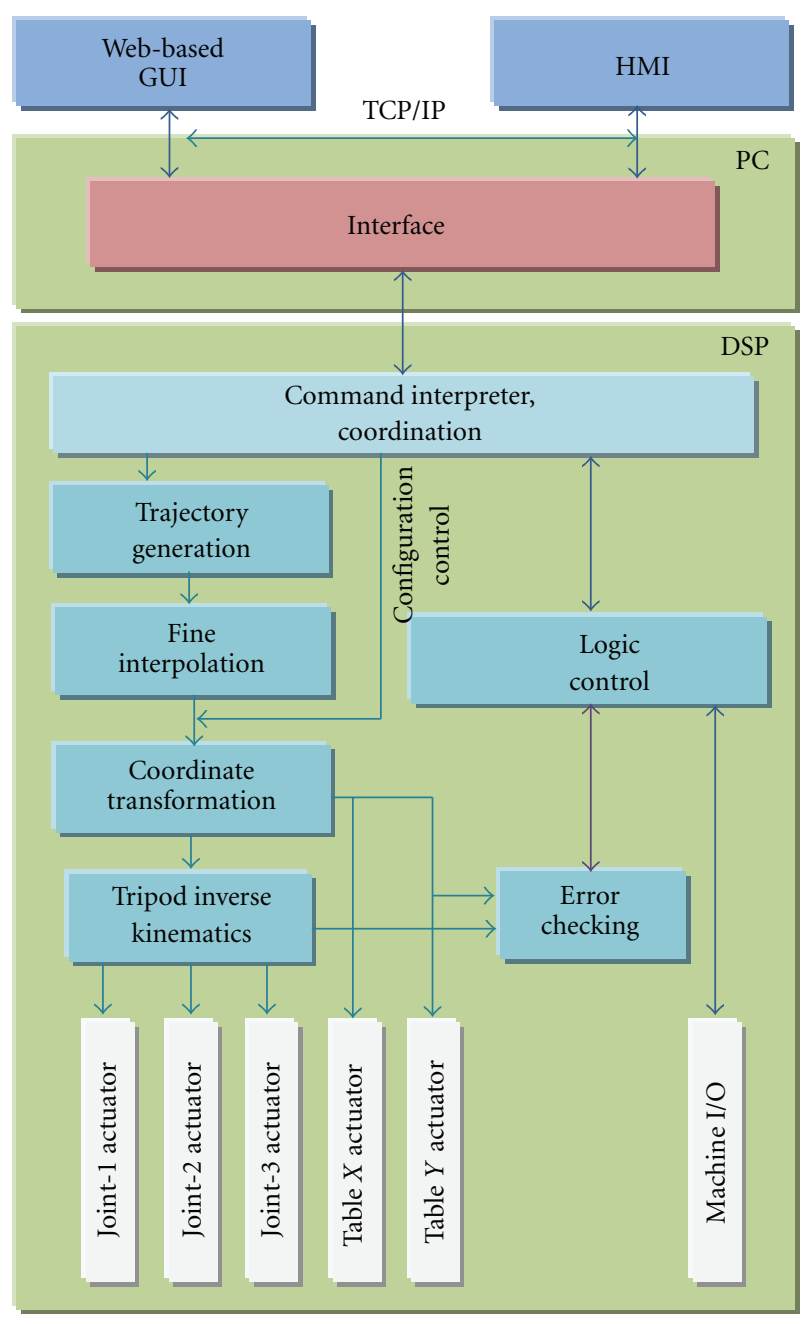

FIGURE 9: Control software architecture.

in real time. Workspace and joint limits are verified against predetermined limits to prevent damage on the machine tool. In case of over travel, the servo system is disabled, and the operator is requested to recover from the error state.

The human machine interface (HMI) functionality is implemented on the PC controller. The user interface itself is a stand-alone program and exchanges information with the OAC through standard TCP/IP communication. The webbased design allows for various HMI implementations at different locations, realizing remote monitoring and control. The inclusion of a forward kinematic solver gives this tool an advantage over other HMI systems for the PKMs, as it can work in two different modes; one is based on the feedback of Cartesian sensors, and the other is based on the feedback of the joint sensors.

\section{Summary}

The proposed reconfigurable machining system includes a tripod-based PKM module, $X-Y$ table, and a reconfigurable arch. The scale and working volume of this reconfigurable machine tool can be tailored to meet the requirements of a specific application. Methodologies to control this machine tool and quickly calibrate the machine for an accurate operation after reconfiguration have been developed. The tripodbased PKM module has a coupled 3-DOF motion. The endeffector motion has a higher speed, higher precision, and is capable of handling a heavier load. This module itself is very versatile, and it can be used as a multifunctional platform for other applications.

\section{References}

[1] Z. M. Bi and W. J. Zhang, "Modularity technology in manufacturing: taxonomy and issues," International Journal of Advanced Manufacturing Technology, vol. 18, no. 5, pp. 381390, 2001.

[2] Z. M. Bi, S. Y. T. Lang, W. M. Shen, and L. Wang, "Reconfigurable manufacturing systems: the state of the art," International Journal of Production Research, vol. 46, no. 4, pp. 967-992, 2008.

[3] D. Sniegulska-Gradzka, M. Klasztorny, and M. Szefarczyk, "An approximate method for determining static and dynamic stiffness of machine tools with rolling guideways," Archives of Civil and Mechanical Engineering, vol. 4, no. 2, 2004.

[4] Y. Koren, "Will industry adopt PKMs?" Manufacturing Engineering, vol. 122, no. 3, p. 240, 1999.

[5] Y. Koren, U. Heisel, F. Jovane et al., "Reconfigurable manufacturing systems," CIRP Annals, vol. 48, no. 2, pp. 527-540, 1999.

[6] Y. Koren and A. G. Ulsoy, "Reconfigurable machine tools and reconfigurable systems," in Proceedings of the 9th International Machine Tool Engineer's Conference (IMEC '00), pp. 180-189, Tokyo, Japan, 2000.

[7] Y.-M. Moon, Reconfigurable machine tool design: theory and application, Ph.D. dissertation, The University of Michigan, Ann Arbor, Mich, USA, 2000.

[8] R. Katz, Z. Li, and F. Pierrot, "Engineering research center for reconfigurable machining systems: conceptual design of a high-speed drilling machine (HSDM) based on PKM module," ERC/RMS Report 37, College of Engineering, The University of Michigan, Ann Arbor, Mich, USA, 2002.

[9] A. V. Cooke, N. Shankar, L. Jones et al., "Advanced reconfigurable machine for flexible fabrication," in Smart Structures and Materials: Industrial and Commercial Applications of Smart Structures Technologies, vol. 2447 of Proceedings of the SPIE, pp. 102-114, San Diego, Calif, USA, March 1995.

[10] R. Bostelman, A. Jacoff, F. Proctor, T. Kramer, and A. Wavering, "Cable-based reconfigurable machines for large scale manufacturing," in Proceedings of the Japan-USA Symposium on Flexible Automation, Mich, USA, July 2000.

[11] I. Fassi and G. J. Wiens, "Multiaxis machining: PKMs and traditional machining centers," Journal of Manufacturing Processes, vol. 2, no. 1, pp. 1-14, 2000.

[12] Y. Zhao, F. Gao, W. Li, W. Liu, and X. Zhao, "Development of 6-dof parallel seismic simulator with novel redundant actuation," Mechatronics, vol. 19, no. 3, pp. 422-427, 2009.

[13] J. G. Wang, Y. Li, and X. Zhao, "Inverse kinematics and control of a 7-DOF redundant manipulator based on the closed-loop Algorithm," International Journal of Advanced Robotic Systems, vol. 7, no. 4, pp. 1-9, 2010.

[14] Y. Yun and Y. Li, "Design and analysis of a novel 6-DOF redundant actuated parallel robot with compliant hinges for high precision positioning," Nonlinear Dynamics, vol. 61, no. 4, pp. 829-845, 2010. 
[15] J. Liu, Y. Wang, S. Ma, and Y. Li, "Enumeration of the non-isomorphic configurations for a reconfigurable modular robot with square-cubic-cell modules," International Journal of Advanced Robotic Systems, vol. 7, no. 4, pp. 58-68, 2010.

[16] Z. M. Bi, S. Y. T. Lang, P. E. Orban, M. Verner, and D. Zhang, "Integrated design toolbox for tripod-based parallel kinematic machines," ASME Journal of Mechanical Design, vol. 129, no. 8, pp. 799-807, 2007.

[17] Z. M. Bi and S. Y. T. Lang, "Joint workspace of parallel kinematic machines," Robotics and Computer-Integrated Manufacturing, vol. 25, no. 1, pp. 57-63, 2009.

[18] R. P. Paul, Robot Manipulators: Mathematics, Programming and Control, MIT Press, Cambridge, Mass, USA, 1981.

[19] Y. Altintas and N. A. Erol, "Open architecture modular tool kit for motion and machining process control," CIRP Annals, vol. 47, no. 1, pp. 295-300, 1998. 

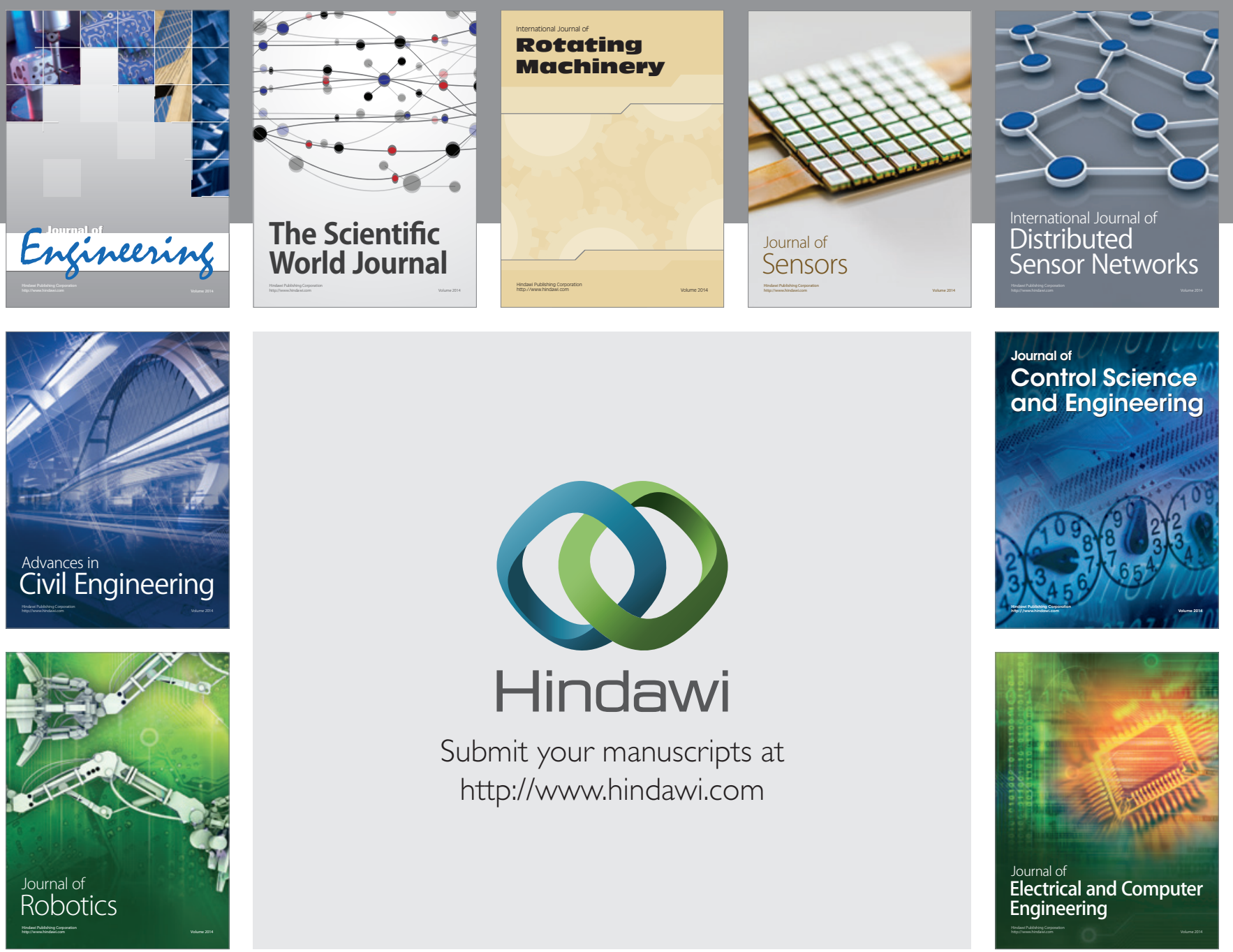

Submit your manuscripts at

http://www.hindawi.com
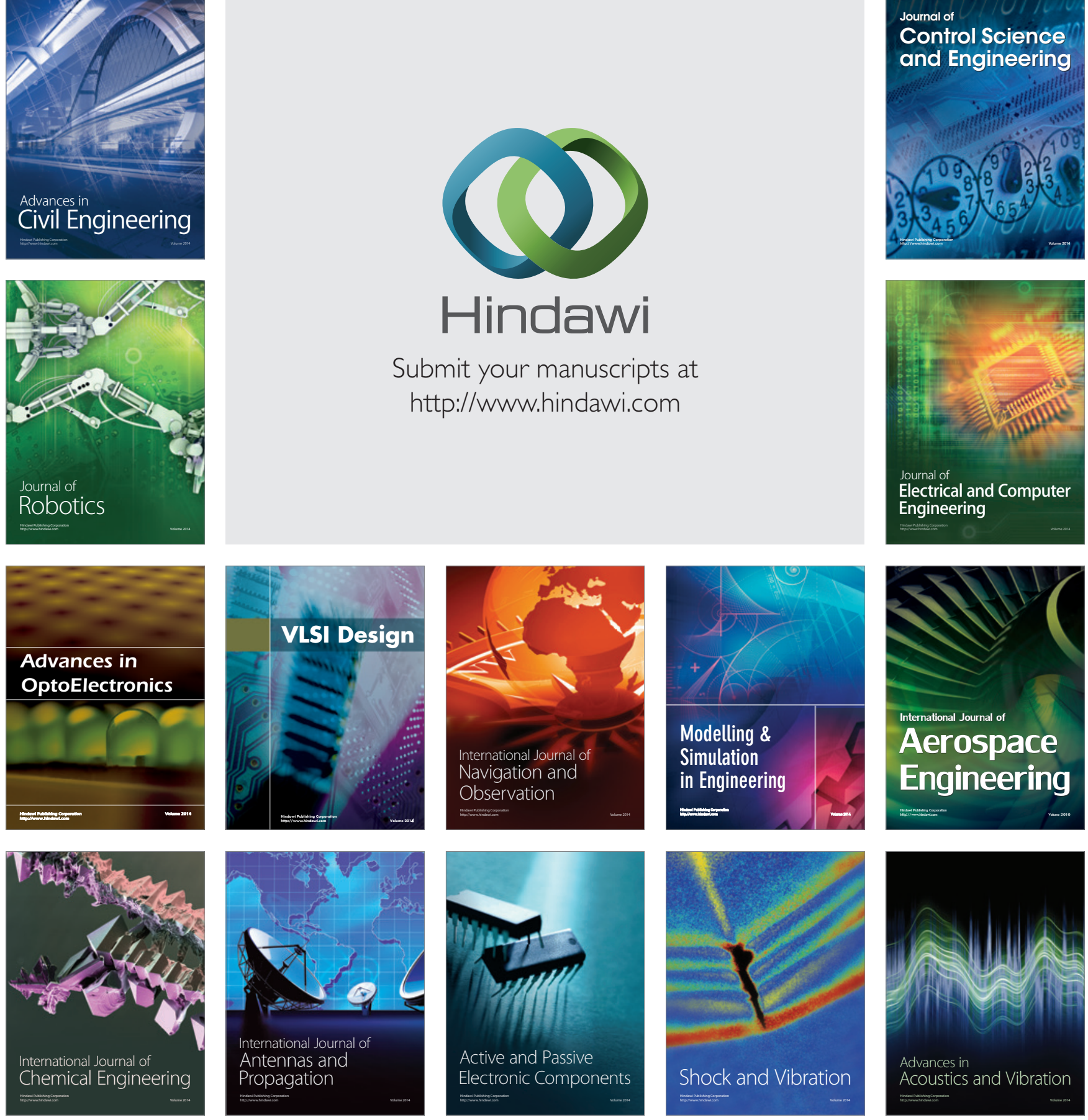